\title{
THE EVENS-KAHN FORMULA FOR THE TOTAL STIEFEL-WHITNEY CLASS
}

\author{
ANDRZEJ KOZLOWSKI
}

\begin{abstract}
Let $G(X)$ denote the (augmented) multiplicative group of classical cohomology ring of a space $X$, with coefficients in $Z / 2$. The (augmented) total Stiefel-Whitney class is a natural homomorphism $w: K O(X) \rightarrow G(X)$. We show that the functor $G($ ) possesses a 'transfer homomorphism' for double coverings such that $w$ commutes with the transfer. This is related to a question of G. Segal. As a special case, we obtain a formula for the total Stiefel-Whitney class of a representation of a finite group induced from a (real) representation of a subgroup of index 2, which is analogous to the one obtained by Evens and Kahn for the total Chern class.
\end{abstract}

1. Introduction. In $[4] \mathrm{L}$. Evens and D. Kahn obtained an interesting formula for the total Chern class of the representation of a finite group induced from a (complex) representation of a subgroup of prime index $p$ (generalizing an earlier formula due to Evens [3]). The purpose of this note is to prove an analogous formula for the total Stiefel-Whitney class of the representation of a finite group induced from a (real) representation of a subgroup of index two. In fact, we formulate our statement in the contex of homotopy theory, thus obtaining an interesting reformulation of a question of G. B. Segal [9].

Let $X$ be a CW-complex and let $G(X)$ denote the group of units of the commutative ring $\prod_{i \geq 0} H^{i}(X ; Z / 2)$. Let

$$
\hat{G}(X)=H^{0}(X ; Z) \oplus G(X) .
$$

This has a natural structure of a commutative ring (as in $[\mathbf{5}]$ ) and the augmented total Stiefel-Whitney class can be viewed as a ring homomorphism $K O(X) \stackrel{w}{\rightarrow}$ $\hat{G}(X)$. Recall that the functor $K O(X)$ has a natural transfer homomorphism for finite coverings, [1 and 6]. Our theorem can be stated as follows.

THEOREM 1.1. The functor $\hat{G}(X)$ admits a natural 'transfer homomorphism' for 2-coverings such that the total Stiefel-Whitney class commutes with the transfer, i.e. the diagram

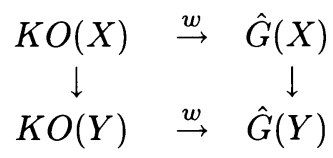

is commutative, where $p: X \rightarrow Y$ is a 2-covering and the vertical maps are the two transfer homomorphisms.

Received by the editors May 24, 1983.

1980 Mathematics Subject Classification. Primary 55N20, 55R40, 55P47, $20 \mathrm{J06.}$

Key words and phrases. Generalized cohomology theory, transfer homomorphism, total StiefelWhitney class.

(C)1984 American Mathematical Society $0002-9939 / 84 \$ 1.00+\$ .25$ per page 
Since the transfer in $\hat{G}(X)$ will be defined by an explicit formula, considering the covering $B H \rightarrow B G$ (where $H$ has index 2 in $G$ ) gives the Evens-Kahn formula.

In [9] G. Segal shows that there is a connective cohomology theory $G^{*}(X)$ with $G^{0}(X)=G(X)$. He also asks if there is a cohomology theory $\hat{G}^{*}(X)$ with $\hat{G}^{0}(X)=$ $\hat{G}(X)$, and such that the total Stiefel-Whitney class extends to a transformation of cohomology theories. In [10] Snaith showed that this is false if we take $\hat{G}^{*}(X)=$ $H^{*}(X ; Z) \oplus G^{*}(X)$, however, this does not mean that there cannot be a different $\hat{G}^{*}(X)$ related to $G^{*}(X)$ in some more complex way. Now, it is well known $[\mathbf{6}]$ that every generalized cohomology theory admits a transfer homomorphism for finite coverings such that transformations of cohomology theories commute with the tranfer. We can ask: does the transfer in $\hat{G}(X)$, defined here for 2-coverings, extend to a transfer defined for all finite coverings and possessing all the usual properties of a transfer homomorphism (e.g. see [8])? If so, then it follows that $w$ commutes with the transfer, thus providing strong support for the affirmative answer to Segal's question.

2. Multiplicative transfers. In this section we shall define the 'transfer homomorphism' in $\hat{G}(X)$ which appears in the statement of Theorem 1.1. Our definition will be based on the results of $[\mathbf{9}]$-the alternative approach is as in [2]. The two approaches are equivalent by $[\mathbf{7}]$.

Let $G(X)$ be as in the Introduction. By [9] there is a cohomology theory $G^{*}(X)$ with $G^{0}(X)=G(X)$. By $[6]$ every generalized cohomology theory possesses a transfer homomorphism for finite coverings; in the above case it shall be called the Segal transfer. Explicitly, the Segal transfer is defined as follows. Let $K_{q}=$ $S^{q} \otimes(Z / 2)$ as in [9]. Then $K=\left\{K_{q}\right\}_{q \geq 0}$ is a graded topological $Z / 2$-module. Let $M(q, n)$ denote the space of maps $K_{q} \times \cdots \times K_{q} \rightarrow K_{n q}$ which are $n$-linear over $Z / 2$ and induce the $n$-fold cup product on homotopy groups. Let $M(n)$ denote the space of graded $n$-linear maps $K \times \cdots \times K \rightarrow K$ which induce the graded cup product on homotopy groups. It now follows from [9] that $M(q, n)$ and $M(n)$ are contractible. In [7] it is shown that the natural $\Sigma_{n}$ action on $M(q, n)$ and $M(n)$ is free. Hence either space can be taken as a model for $E \Sigma_{n}$. We can now define a map $D: G^{0}(X) \rightarrow G^{0}\left(M(n) \times_{\Sigma_{n}} X^{n}\right)$ as follows. Let $G=\bigoplus_{i>1} K(Z / 2 ; i)$ represent $G(X)$ (see [6]). Let $f: X \rightarrow G$ represent $[f] \in G(X) . D$ is defined by the formula $D([f])=[D(f)]$, where $D(f): E \Sigma_{n} \times_{\Sigma_{n}} X^{n} \rightarrow G$ is given by

$$
D(f)\left(\alpha, x_{1}, \ldots, x_{n}\right)=\alpha\left(f\left(x_{1}\right), \ldots, f\left(x_{n}\right)\right) .
$$

For every $n$-covering $X \rightarrow Y$ we have a map $\phi: Y \rightarrow E \Sigma_{n} \times_{\Sigma_{n}} X^{n}$ unique up to homotopy, called the pretransfer [5]. The Segal transfer is defined by $N=$ $\phi^{*} D: G(X) \rightarrow G(Y)$, where we identify $E \Sigma_{n}$ with $M(n)$. Since $N$ is the transfer in a generalised cohomology (Segal's cohomology) it must possess all the usual properties.

There is also another, closely related 'transfer', defined for elements of $H^{i}(X ; Z / 2)$ and taking values in $H^{n i}(Y ; Z / 2)$ (this 'transfer' has no obvious interpretation in terms of generalized cohomology theories). ${ }^{1}$ In detail, we define a

\footnotetext{
${ }^{1}$ However, see J. P. May, $H_{\infty}$ ring spectra and their applications, Proc. Sympos. Pure Math., vol. 32, Amer. Math. Soc., Providence, R.I., 1978, p. 242
} 
map, denoted also by $D$,

$$
D: H^{i}(X ; Z / 2) \rightarrow H^{n i}\left(E \Sigma_{n} \times \Sigma_{n} X^{n} ; Z / 2\right),
$$

where this time we identify $E \Sigma_{n}$ with $M(i, n)$ by the same formula as above with the obvious reinterpretation of symbols. Just as above, we define $N: H^{i}(X ; Z / 2) \rightarrow$ $H^{n i}(Y ; Z / 2)$ by $N=\phi^{*} D$. It is clear that $N$ is multiplicative, in the sense that $N(x y)=N(x) N(y)$ for $x \in H^{i}(X ; Z / 2)$ and $y \in H^{k}(X ; Z / 2)$.

Now let $X \rightarrow Y$ be a 2 -covering. We define a 'transfer' $\hat{N}: \hat{G}(X) \rightarrow \hat{G}(Y)$ as follows. Let $\hat{D}: G(X) \rightarrow G\left(E \Sigma_{2} \times_{\Sigma_{2}} X^{2}\right)$ be given by the formula

$$
\begin{aligned}
\hat{D}\left(m, 1+x_{1}+\cdots+x_{n}+\cdots\right)=( & 2 m, D\left(1+x_{1}+\cdots+x_{n}+\cdots\right) \\
& \left.+\sum_{i=1}^{\infty} D\left(x_{i}\right)\left((1+t)^{m-i}+1\right)+(1+t)^{m}+1\right),
\end{aligned}
$$

where $D$ is defined above and $t$ is the element of $H^{1}\left(E \Sigma_{2} \times_{\Sigma_{2}} X^{2} ; Z / 2\right)$ which is the image of the nonzero element of $H^{1}(B Z / 2 ; Z / 2)$. The augmented transfer for 2-coverings $\hat{N}$ is now defined by $\hat{N}=\phi^{*} D$, where $\phi$ is the pretransfer as above.

We have

PROPOSITION 2.1. $\hat{N}$ is a homomorphism, natural with respect to pull-backs of 2-coverings.

To prove this proposition we need some lemmas. Let $E \Sigma_{2} \times X \rightarrow E \Sigma_{2} \times X^{2}$ be given by $(e, x) \mapsto(e, x, x)$. This is a $\Sigma_{2}$-equivariant map, and hence induces a map $\Delta: B \Sigma_{2} \times X \rightarrow E \Sigma_{2} \times \Sigma_{2} X^{2}$. Let $i: X^{2} \rightarrow E \Sigma_{2} \times_{\Sigma_{2}} X^{2}$ be the inclusion $(x, x) \mapsto\left[\left(e_{0}, x, x\right)\right]$, where $e_{0}$ is some chosen base point in $E \Sigma_{2}$.

LEMMA 2.2 .

$$
\Delta^{*} \oplus i^{*}: H^{*}\left(E \Sigma_{2} \times_{\Sigma_{2}} X^{2} ; Z / 2\right) \rightarrow H^{*}\left(B \Sigma_{2} \times X ; Z / 2\right) \oplus H^{*}\left(X^{2} ; Z / 2\right)
$$

is injective.

ProOF. See $[\mathbf{1 1}]$.

LEMMA 2.3. For the covering $X \simeq E \Sigma_{2} \times X \rightarrow B \Sigma_{2} \times X$ the pretransfer is given by (the homotopy class of) $\Delta$.

ProOF. Easy. For details see [7].

LEMMA 2.4. For the covering $X \simeq E \Sigma_{2} \times X \rightarrow B \Sigma_{2} \times X$ the multiplicative transfers defined above are given by

(i) $N\left(1+x_{1}+\cdots+x_{k} \cdots\right)=1+\sum_{i, k} \mathrm{Sq}^{i}\left(x_{k}\right) t^{k-i}$,

(ii) $N\left(x_{k}\right)=\sum_{i} \mathrm{Sq}^{i}\left(x_{k}\right) t^{k-i}$,

(iii) $\hat{N}\left(m, 1+x_{1}+\cdots\right)=\left(2 m,(1+t)^{m}+\sum_{i} N\left(x_{i}\right)(1+t)^{m-i}\right)$.

PROOF. See [7].

PROOF OF PROPOSITION 2.1. It is clear that $\hat{N}$ is natural for pull-backs of 2 coverings. From Lemma 2.4(iii) we can easily check that $\hat{N}$ is a homomorphism for the covering $X \rightarrow B \Sigma_{2} \times X$. To prove that $\hat{N}$ is a homomorphism for all 2-coverings 
it is enough to show that $\hat{D}: \hat{G}(X) \rightarrow \hat{G}\left(E \Sigma_{2} \times_{\Sigma_{2}} X^{2}\right)$ is a homomorphism. By Lemma 2.2 it suffices to prove that $\Delta^{*} \hat{D}: \hat{G}(X) \rightarrow \hat{G}\left(B \Sigma_{2} \times X\right)$ and $i^{*} \hat{D}: \hat{G}(X) \rightarrow$ $\hat{G}\left(X^{2}\right)$ are homomorphisms. However, the former follows from Lemma 2.3 and the above, and the latter is obvious from the definition of $\hat{D}$.

Now let $F^{*}(X)$ be any generalized cohomology theory. If $X \rightarrow Y$ is a finite covering, let $\operatorname{tr}_{F}: F^{0}(X) \rightarrow F^{0}(Y)$ denote the Kahn-Priddy transfer.

Proposition 2.5. Let $g: F^{0}(X) \rightarrow \hat{G}(X)$ be a natural homomorphism. Then $g$ commutes with the transfer for all 2-coverings i.e. $\left.g\left(\operatorname{tr}_{F}(x)\right)=\hat{N}(g(x))\right)$ if and only if $g$ commutes with the transfer for the coverings $X \rightarrow B \Sigma_{2} \times X$.

PROOF. From the definition of the transfer in a generalized cohomology theory in $[6]$ and from the definition of $\hat{N}$ it follows that we only need to show that the diagram

$$
\begin{array}{ccc}
F^{0}(X) & \stackrel{D_{F}}{\rightarrow} & F^{0}\left(E \Sigma_{2} \times_{\Sigma_{2}} X^{2}\right) \\
g \downarrow & & \downarrow g \\
G(X) & \stackrel{\hat{D}}{\rightarrow} & G\left(E \Sigma_{2} \times_{\Sigma_{2}} X^{2}\right)
\end{array}
$$

commutes, where $D_{F}$ is induced by the Dyer-Lashof map for $F$ and $\hat{D}$ is as above. However, this is clear from Lemmas 2.2 and 2.3.

3. Proof of Theorem 1.1. Recall that the augmented total Stiefel-Whitney class is a natural ring homomorphism $w: K O(X) \rightarrow \hat{G}(X)$, which takes the real vector bundle $E$ to $\left(\operatorname{dim} E, 1+w_{1}(E)+w_{2}(E)+\cdots\right)$. By Proposition 2.5 it suffices to prove that the total Stiefel-Whitney class commutes with the transfer for coverings of the form $X \rightarrow B \Sigma_{2} \times X$, for all spaces $X$. Using the naturality of $\hat{N}$ and the fact that it is a homomorphism (Proposition 2.1) we easily see that the splitting principle in real $K$-theory implies that we only need to show that $w \operatorname{tr}_{K O}(x)=\hat{N} w(x)$ for a line bundle $x$ over $X$. Suppose $x$ has the first Stiefel-Whitney class $w_{1}(x)$. Then $\operatorname{tr}_{K O}(x) \in K O\left(B \Sigma_{2} \times X\right)$ is the sum of two line bundles with first Stiefel-Whitney classes $w_{1}(x)$ and $w_{1}(x)+t$, where as above $t$ denotes the image of the nonzero element of $H^{1}\left(B \Sigma_{2} ; Z / 2\right)$. Hence we have

$$
\begin{aligned}
w \operatorname{tr}_{K O}(x) & =\left(2,\left(1+w_{1}(x)\right)\left(1+w_{1}(x)+t\right)\right) \\
& =\left(2,1+t+w_{1}(x)^{2}+t w_{1}(x)\right) .
\end{aligned}
$$

On the other hand, from Lemma 2.4 we obtain

$$
\begin{aligned}
\hat{N} w(x) & =\hat{N}\left(1,1+w_{1}(x)\right)=\left(2,1+\operatorname{Sq}^{0}\left(w_{1}(x)\right) t+\operatorname{Sq}^{1}\left(w_{1}(x)\right)+t\right) \\
& =\left(2,1+w_{1}(x) t+w_{1}(x)^{2}+t\right) .
\end{aligned}
$$

This completes the proof.

\section{REFERENCES}

1. M. F. Atiyah, Characters and cohomology of finite groups, Inst. Hautes Études Sci. Publ. Math. 9 (1961), 23-64.

2. L. Evens, A generalization of the transfer map in the cohomology of groups, Trans. Amer. Math. Soc. 108 (1963), 54-65.

3. 180-193. 
4. L. Evens and D. Kahn, Chern classes of certain representations of symmetric groups, Trans. Amer. Math. Soc. 245 (1978/79), 309-330.

5. A. Grothendieck, La théorie des classes de Chern, Bull. Soc. Math. France 86 (1958), 137-154.

6. D. Kahn and S. B. Priddy, Applications of the transfer to stable homotopy theory, Bull. Amer. Math. Soc. 78 (1972), 981-987.

7. A. Kozlowski, The transfer in Segal's cohomology, Illinois J. Math. (to appear)

8. F. Roush, Thesis, Princeton University, 1971.

9. G. B. Segal, The multiplicative group of classical cohomology, Quart. J. Math. Oxford Ser. 26 (1975), 289-293.

10. V. Snaith, The total Chern and Stiefel-Whitney classes are not infinite loop maps, Illinois J. Math. 21 (1977), 300-303.

11. D. G. Quillen, The Adams conjecture, Topology 10 (1970), 67-80.

817-1 AzUma 2 Chome, SAKURA-MURA, Nilhari-Gun, IbARAKI, JAPAN 